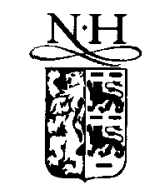

ELSEVIER

\title{
Supersaturation and crystal growth in gas anti-solvent crystallization
}

\author{
Clifford Y. Tai*, Chuen-Song Cheng \\ Department of Chemical Engineering, National Taiwan University, Taipei 106, Taiwan, ROC
}

Received 25 August 1997

\begin{abstract}
A technique of developing a low expansion rate of solution under constant temperature was employed to examine the saturation behavior of expanded solutions and the growth of crystals in the gas anti-solvent crystallization. When solutions were expanded under pressure, three types of saturation behavior were observed. The behaviors are explicable using an $\alpha$ factor. The growth phenomenon, growth rate, and growth mechanism of crystals in the GAS process were similar to those usually found in the conventional processes of solution crystallization except that millimeter-sized inorganic crystals without well-defined faces cannot re-facet themselves. C 1998 Elsevier Science B.V. All rights reserved.
\end{abstract}

Keywords: Gas anti-solvent crystallization; Expansion; Supersaturation; Crystal growth

\section{Introduction}

The conventional salting-out process is economically inefficient as compared with other crystallization processes, because it requires a separation unit to recover the precipitant, which is usually a solvent or a liquid. Recently, gases at elevated pressure are found to be soluble in many organic solvents, and then to expand the solvents and reduce the solvent power of dissolving solid. This process is known as the gas anti-solvent (GAS) crystallization, using a compressed gas as the precipitant. The GAS process removes the obstacle of economic

\footnotetext{
* Corresponding author. Fax: + 88623623040
}

inefficiency for recovering the precipitant, because the dissolved gas can be recovered easily by a simple pressure releasing. It also takes advantage of the fact that pressure can be readily programmed to make high-quality crystals $[1,2]$ and to accomplish fractional crystallization [3].

Gallagher et al. [1] first explored the GAS process and reported three findings: (1) Individual crystals of regular shape were nucleated at low expansion rates and low expansion levels; (2) While nitroguanidine was readily crystallized in a wide spectrum of operating conditions, naphthalene crystals was obtained only in a very narrow range of pressure and temperature; (3) Equilibrium expansion occurred within a few seconds under a given set of operating conditions. 
After the publication of the pioneer work, investigations on various aspects of the GAS process have been conducted. A few authors measured and modeled the solute solubilities of the crystallization system [3-5]. Others demonstrated the applicability of the process to separate mixed solutes [6-9] and to control crystal size distribution $[2,10]$. Recently, Tai and Cheng [11] studied the pressure effects of carbon dioxide on the expansion level and saturation behavior of various solutions saturated at atmospheric pressure. However, fundamental work on crystal growth is lacking.

This study is a continuous work of Tai and Cheng [11] to investigate the saturation behavior of solutes, and the growth of crystals in the GAS process. Carbon dioxide was used to expand a number of solutions with a technique of low expansion rates, i.e. $\mathrm{CO}_{2}$ was introduced above the liquid and let it diffuse into the saturated solution. Higher rates of expansion, either by stirring the liquid or by bubbling $\mathrm{CO}_{2}$ through the solution, which caused a rapid rise in temperature, were excluded from this report. The distinct types of solute saturation behavior caused by solution expansion were explained, and the growth phenomena of nucleated crystals and introduced seeds were interpreted with existing growth theories in the literature. Also, growth rates were measured, and compared with those from aqueous solutions.

\section{Experimental procedure}

In this experiment, various solutes and solvents were selected to prepare the solutions, including organic, metal-organic, and inorganic compounds for solutes, and acetone, ethanol, and ethanolwater mixtures for the solvent. The use of methanol-water mixture allows the investigation of many solids that are only sparingly soluble in pure organic solvents.

The apparatus is schematically shown in Fig. 1, which is essentially the same as that used by Tai and Cheng [11]. It was designed for observing the solution expansion and solute saturation behaviors, and for measuring the expansion level and crystal growth rates during the expansion courses.

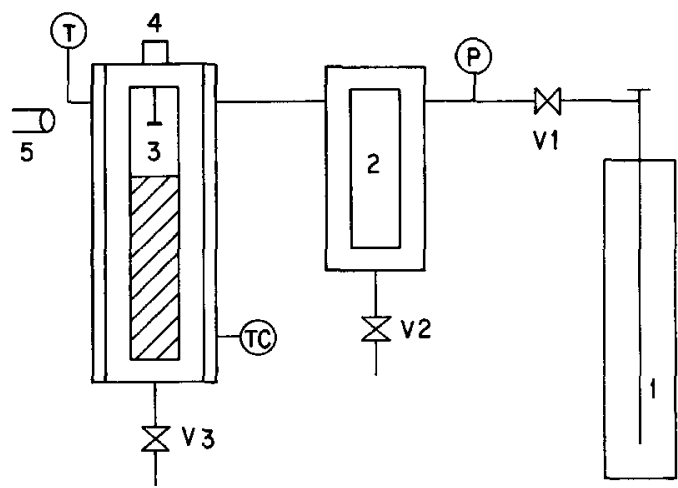

Fig. 1. Experimental apparatus. (1) Carbon dioxide cylinder; (2) Overflow cell; (3) Crystallization cell; (4) Seed-fixing needle; (5) Microscope; P: Pressure indicator, T: Temperature indicator, $\mathrm{TC}$ : Temperature controller, $\mathrm{V}_{1}$ : Needle valve, $\mathrm{V}_{2}$ : Ball valves, $V_{3}$ : Ball valves.

The procedure of conducting an experimental run began by loading the cell with a saturated solution of 120 or $100 \mathrm{ml}$, depending on the expansion ratio, and inserting a seed crystal into it. The saturated solution was prepared at room temperature and atmospheric pressure, with the solubilities determined by the gravimetric method. The seed, either a single crystal of 0.5 to $1.0 \mathrm{~mm}$ or a compressed powder, came directly from the purchased chemicals without recrystallization. After loading the solution and inserting the seed, carbon dioxide ( $99 \%$ pure) was introduced into the cell above the top of the saturated solution to elevate the system pressure to a desired level, while the crystallization cell was kept at room temperature. Then, the solution was allowed to expand gradually by absorbing carbon dioxide. By this way, the solution temperature would be able to remain constant and the saturation behavior of solution due to expansion alone can be identified. During the expansion course, the volume of the solution was recorded with time to study the expansion profile and the seed was photographed to examine its change when it became immersed in the expanded solution. An experimental run was normally stopped after the expansion process reached equilibrium, or the seed crystal dissolved entirely, depending on the solubility behavior of the system. The chemicals used in this study were purchased 
from various sources, with all of them being reagent grade.

\section{Results and discussion}

The expansion levels and saturation behaviors of a saturated solution caused by the compressed carbon dioxide have been reported by Tai and Cheng [11]. A solution saturated at atmospheric pressure may become highly, moderately, or negatively supersaturated when it is expanded to various levels at a slow rate, depending on the types of solvent and solute. As a result, solutes may precipitate by catastrophic nucleation from bulk solutions or by heterogeneous nucleation on container-walls, or they may not even precipitate at all. Generally, most inorganic solutes precipitate by the heterogeneous nucleation and a few by the catastrophic nucleation; metal-organic solutes may precipitate either by the heterogeneous nucleation or by the catastrophic nucleation; and organic solutes may precipitate by the heterogeneous nucleation or may not precipitate at all. For example, the $\mathrm{NaCH}_{3} \mathrm{COO} /$ ethanol and $\mathrm{LiCl} /$ acetone systems belong to the catastrophic nucleation system; the acetominophen/ethanol, fructose/ethanol, $\mathrm{NH}_{4} \mathrm{Cl}$ / ethanol, and $\mathrm{NaSCN} /$ ethanol systems belong to the heterogeneous nucleation or growth system; the adipic acid/ethanol and naphthalene/toluene systems belong to the undersaturation system. The classification of more solution systems is shown elsewhere [11]. Most of the expansion levels are between 10 and $30 \%$ of the original volume, under the carbon dioxide pressure below 61 bar.

The diversity of supersaturation levels caused by $\mathrm{CO}_{2}$ expansion can be explained by considering the supersaturation of a solution originally saturated at atmospheric pressure. The supersaturation can be expressed as

$$
\begin{aligned}
\Delta C & =C_{0}-C_{\mathrm{e}} \\
& =C_{0}-\left[C_{\mathrm{e} 0}+\int_{0}^{C_{\mathrm{g}}}\left(\partial C_{\mathrm{e}} / \partial C_{\mathrm{g}}\right)_{T, P} \mathrm{~d} C_{\mathrm{g}}\right],
\end{aligned}
$$

where $C_{0}$ is the solute solubility at atmospheric pressure, $C_{\mathrm{e} 0}$ the solute solubility at an elevated pressure but with zero concentration of dissolved carbon dioxide, $C_{\mathrm{e}}$ the solute solubility in the expansion course. $C_{0}, C_{\mathrm{e} 0}$, and $C_{\mathrm{e}}$ are on a carbon dioxide free basis. $C_{\mathrm{g}}$ is the concentration of dissolved carbon dioxide on a solute-free basis. Assuming that $C_{\mathrm{co}}$ departs little from $C_{0}$ at moderate pressures, Eq. (1) becomes

$\Delta C=\int_{0}^{C_{\mathrm{g}}}-\left(\partial C_{\mathrm{e}} / \partial C_{\mathrm{g}}\right)_{T, P} \mathrm{~d} C_{\mathrm{g}}=\int_{0}^{C_{\mathrm{g}}} \alpha \mathrm{d} C_{\mathrm{g}}$,

where $\alpha$ is defined as $-\left(\partial C_{e} / \partial C_{\mathrm{g}}\right)_{T, P}$. Eq. (2) shows that the supersaturation is controlled by the $\alpha$ factor. The nucleation, growth, and undersaturation systems must have a large, moderate, and negative $\alpha$ values, so that they were accompanied by a high, moderate, and negative supersaturation respectively when they were expanded.

The diversity of $\alpha$ values among the three groups of systems can be understood qualitatively by speculating on the complex thermodynamic interaction among the solvent, solute, and carbon dioxide of an expanded solution. Because the $\alpha$ factor physically means the change of solute concentration with the dissolution of carbon dioxide for the solution to maintain equilibrium, its value is affected in two ways. One is that the dissolved carbon dioxide reduces some of solvent dissolving power by decreasing the partial molar volume of the solvent [4]. The other is that the dissolved carbon dioxide itself becomes condensed and acts as an additional solvent for the solute. If the solute is insoluble in carbon dioxide, the solution will have a positive $\alpha$. This gives the nucleation and growth system. In contrast, if the solute is soluble in carbon dioxide, the solution will have a negative $\alpha$ when the increasing amount of carbon dioxide dissolving power surpasses the reduced amount of solvent dissolving power. This case is of undersaturation system. The nucleation systems have large $\alpha$ values probably because their solutes are insoluble in condensed carbon dioxdide and the sensitivity of solvent-solute dissolving power to the concentration of dissolved carbon dioxide in the systems is high. The growth systems have moderate $x$ values perhaps for the same reasons as is given for the nucleation systems except that now the sensitivity is moderate. These conclusions are supported by 
the solubility data of condensed carbon dioxide in the literature. For example, lithium chloride, which is a solute of the nucleation systems, and urea, which is a solute of the growth systems, are insoluble in the condensed carbon dioxide, whereas naphthalene, which is a solute of the undersaturation systems, is soluble in the condensed carbon dioxide [12].

The growth phenomena of several single-crystal seeds have been studied. The organic seeds exhibited the same growth phenomena as those usually found in the crystal growth from liquid solutions. The acetaminophen seed without well-defined faces, for example, finally developed into a $2 \mathrm{~mm}$ polyhedron that is bounded by light-reflecting faces in ethanol solution as shown in Fig. 2. The sucrose seed in a mixed-solution of methanol and water has the same growth phenomena as acetaminophen. In contrast, the inorganic seeds showed abnormal growth phenomena which are seldom found in conventional solution growth. For example, instead of growing into larger single crystals bounded by well-defined faces the sodium chloride and potassium nitrate seeds without well-defined faces, produced some small polyhedrons and needles, as shown in Fig. 3 for potassium nitrate. It seems that organic crystals of millimeter-size can re-facet in the GAS process, but inorganics cannot.

The habit, face structure, average size, and growth rate of a number of crystals developed from the nuclei precipitated in the expansion course and/or from the compressed-powder seeds are summarized in Table 1 . The habits of the crystals included polyhedrons, plates, needles, whiskers, and dendrites. Each of the organic and metal-organic crystals were of unique habit, e.g., polyhedrons for the sucrose, plates for the anthracene, needles for the urea, whiskers for the pentaerythritol, and dendrites for the monosodium glutamate crystals. In contrast, several of the inorganic crystals were of multiple habits although some others were found only polyhedrons. For example, sodium chlorate crystals and ammonium perchlorate crystals (shown in Fig. 4) produced polyhedrons, plates, needles, and whiskers. These results suggest that tiny crystals in the GAS process may develop into larger ones with a variety of habits, just as in the conventional processes of solution crystallization.

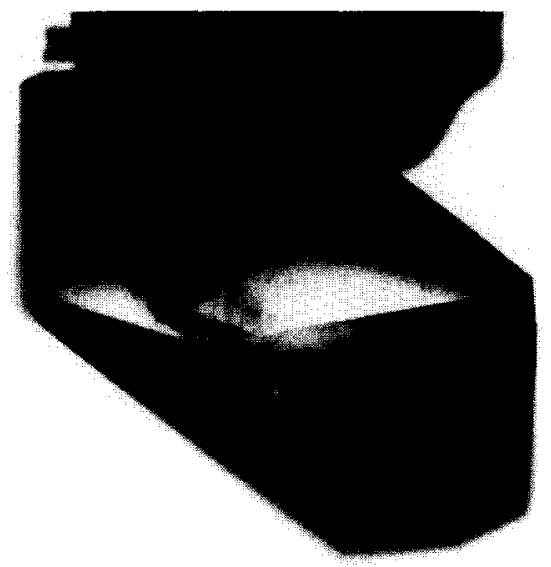

Fig. 2. Polyhedron developed from a single-crystal seed of acetaminophen in ethanol/ $\mathrm{CO}_{2}$ solution.

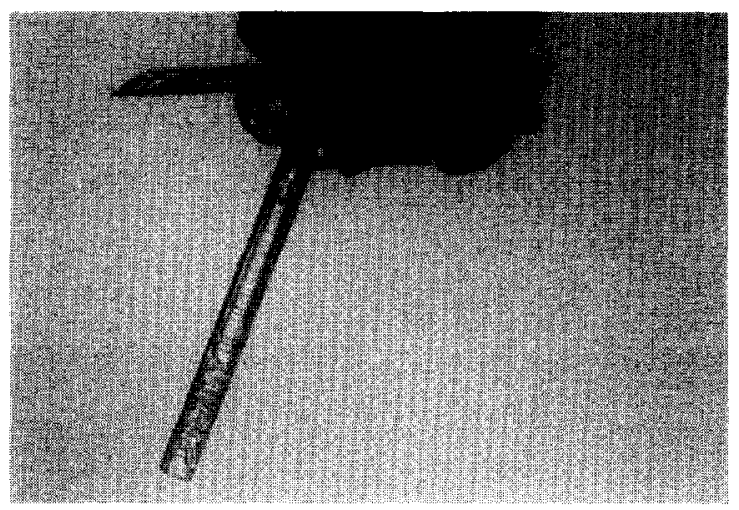

Fig. 3. Abnormal growth of single crystal of potassium nitrate from solution expanded with carbon dioxide.

In spite of the diverse habits, most of the produced crystals were bounded by well-defined faces. For example, organic acetaminophen and sucrose polyhedrons of $0.3 \mathrm{~mm}$ developed normally. Also, inorganic sodium chloride, ammonium chloride, and magnesium sulfate polyhedrons of $0.4,0.2$, and $0.3 \mathrm{~mm}$, respectively, were grown without exhibiting abnormal growth despite their rather large sizes. These results indicate that crystals generally undergo facet-growth in the GAS process, just as crystals usually do in conventional processes of solution crystallization. 
Table 1

Habits, face structures, average sizes, and growth rates of crystals developed from nuclei and/or powder-crystal seeds in the GAS process

\begin{tabular}{|c|c|c|c|c|c|}
\hline Solute & Solvent & Habit & Face struct. ${ }^{\mathbf{b}}$ & $\begin{array}{l}\text { Ave. size } \\
(\mathrm{mm})\end{array}$ & $\begin{array}{l}\text { Growth rate } \\
\left(10^{-8} \mathrm{~m} / \mathrm{s}\right)\end{array}$ \\
\hline Acetaminophen & Ethanol & Polyhedron & W & 0.3 & 1.4 \\
\hline Fructose & Ethanol & Polyhedron & $\mathrm{W}, \mathrm{M}$ & 0.3 & 0.4 \\
\hline Sucrose & Meth $80^{a}$ & Polyhedron & $\mathrm{W}$ & 0.3 & 0.3 \\
\hline Anthracene & Ethanol & Plate & W & 0.6 & 0.9 \\
\hline Urea & Ethanol & Needle & - & 1 & 6.2 \\
\hline Pentaerythritol & Meth60 & Whisker & - & 0.1 & 0.3 \\
\hline Monosodium glutamate & Meth70 & Dendrite & - & 1 & 1.7 \\
\hline $\mathrm{NH}_{2} \mathrm{OH} \cdot \mathrm{HCl}$ & Ethanol & Polyhedton & $\mathrm{W}, \mathrm{M}$ & 0.2 & 0.2 \\
\hline $\mathrm{NH}_{4} \mathrm{Cl}$ & Ethanol & Polyhedron & W & 0.2 & 0.2 \\
\hline $\mathrm{NaCl}$ & Meth80 & Polyhedron & $\mathrm{W}, \mathrm{H}$ & 0.4 & 3.8 \\
\hline $\mathrm{MgSO}_{4} \cdot 7 \mathrm{H}_{2} \mathrm{O}$ & Meth70 & Polyhedron & W & 0.3 & 1.4 \\
\hline \multirow[t]{2}{*}{$\mathrm{NaSCN}$} & Ethanol & Polyhedron & $\mathrm{W}, \mathrm{M}$ & 0.5 & 1.3 \\
\hline & & Plate & W & 1 & 3.3 \\
\hline \multirow[t]{4}{*}{$\mathrm{NaClO}_{3}$} & Ethanol & Polyhedron & W & 0.1 & 0.2 \\
\hline & & Plate & W & 0.3 & 0.3 \\
\hline & & Needle & W & 0.3 & 0.4 \\
\hline & & Whisker & - & 0.4 & 0.5 \\
\hline \multirow[t]{4}{*}{$\mathrm{NH}_{4} \mathrm{ClO}_{4}$} & Ethanol & Polyhedron & W & 0.2 & 1.1 \\
\hline & & Plate & W & 0.5 & 7.8 \\
\hline & & Needle & W & 1 & 10 \\
\hline & & Whisker & - & 1 & 12 \\
\hline
\end{tabular}

${ }^{\mathrm{a}}$ Meth X means methanol-water mixture of $\mathrm{X}$ wt $\%$ methanol.

${ }^{\mathrm{b}} \mathrm{W}$ : well defined; $\mathbf{M}$ : parallel straight macrosteps; $\mathrm{H}$ : hopper faces.

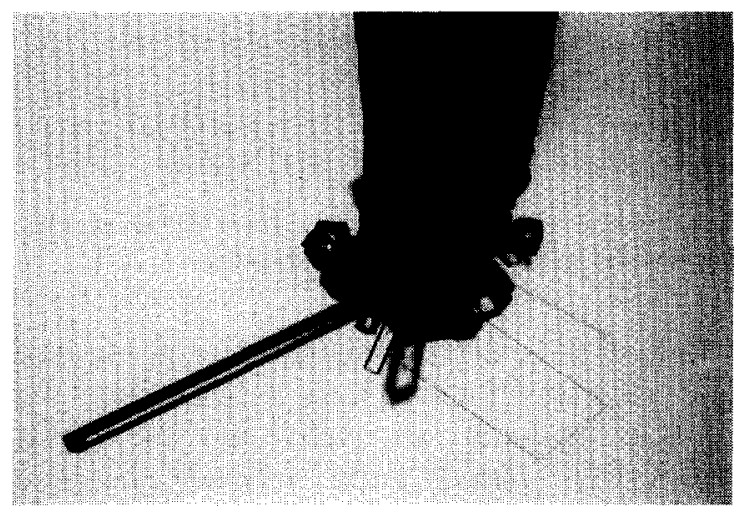

Fig. 4. Various crystal habits of ammonium perchlorate growing on a single seed from ethanol/ $\mathrm{CO}_{2}$ solution.

Although most of the well-defined faces were flat, two particular structures were occasionally found. First, hopper faces were observed on a few sodium chloride crystals. Second, parallel and straight macrosteps were seen on the fructose, hydroxylamine hydrochloride, and sodium thiocyanate (Fig. 5) seed crystals. These structures imply the crystal growth mechanism in the GAS process, which will be discussed later.

The growth rates of the various crystals in Table 1 ranged from 0.2 to $1.2 \times 10^{-7} \mathrm{~m} / \mathrm{s}$. Notable is that the growth rates are of the same order of magnitude as those from aqueous solutions [13]. Also worth attention is that the polyhedral crystals generally grew more slowly than the crystals with other habits; e.g., the polyhedral ammonium perchlorate crystals grew only one tenth as fast as the needle ones. This fact is also indicative of the mechanisms of crystal growth in the GAS process, which is to be discussed in the following sections.

The controlling mechanisms of crystal growth in the GAS process can be deduced from the growth rates and growth phenomena by applying the twostep model of crystal growth [14-16]. According to 


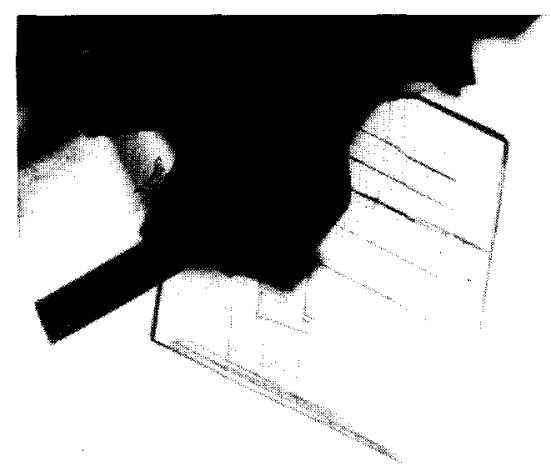

Fig. 5. Cross macrosteps on a grown $\mathrm{NaSCN}$ seed from ethanol/ $\mathrm{CO}_{2}$ solution.

this model, the crystal-growth process is composed of a surface-integration step connected in series with a bulk-diffusion step. In stagnant solutions, different crystallographic faces of crystals show various growth rates because they encounter similar bulk-diffusion but different surface-integration barriers. Crystal faces under integration-control condition will grow much more slowly than those with minor surface-integration barriers, i.e., in the diffusion-control condition. Therefore, the fact that the polyhedral ammonium perchlorate crystals grew only one tenth as fast as the needle ones suggests that the polyhedrons were grown in the integration-control condition. In addition, the dendritic habit of monosodium glutamate crystals, shown in Fig. 6, implies that the crystals were grown in the diffusion-control condition because only in such condition can crystals be found dendritic [17]. In summary, crystal growth in the GAS process may be controlled either by the surfaceintegration step or by the bulk-diffusion step, just like crystal growth in liquid solution.

In addition to distinguish the controlling step, several surface-integration mechanisms can be inferred from the growth phenomena by the existing crystal-growth theories in the literature. First, the multiple habits of ammonium perchlorate and sodium chlorate crystals indicate the spiral mechanism according to Strickland-Constable [18] and Lewis [19]. Second, the hopper growth of sodium chloride crystals implies the mechanism of twodimensional nucleation near the corners of crystal

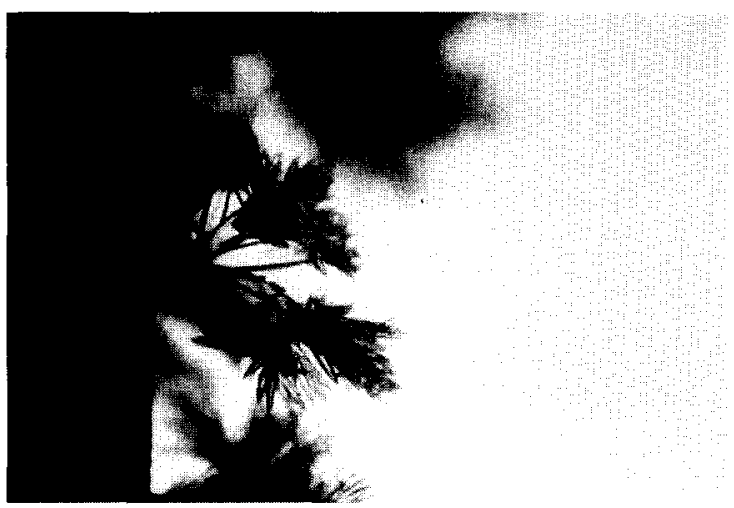

Fig. 6. Dendritic growth of monosodium glutamate crystal on the glass wall of growth cell.

faces in light of the stability theory of crystal growth from solutions [20]. Last, the parallel and straight macrosteps on the faces of fructose, sodium thiocyanate, and hydroxylamine hydrochloride crystals suggest the mechanism of two-dimensional nucleation near the edge of crystal faces [21]. Therefore, crystal faces exposed in the environment of a GAS process may integrate by the spiral mechanism or by the two-dimensional nucleation mechanism, similar to the conventional processes of solution crystallization.

\section{Conclusions}

The experimental technique employed in this research provides a convenient means for finding the expansion profile of solutions, for investigating the saturation behavior of solutes, and for studying the crystal growth in the GAS process.

Solutions saturated atmospherically may become highly, moderately, or negatively supersaturated when they are expanded to low levels at low rates. As a result, solutes may precipitate by catastrophic nucleation from bulk solutions or by heterogeneous nucleation on container-walls, or they may even not precipitate at all. The diversity of the supersaturation levels can be explained by using an $\alpha$ factor.

Crystals show a variety in habit and undergo facet-growth in the GAS process. Their growth rates are mostly around $10^{-8} \mathrm{~m} / \mathrm{s}$. Their growth 
process may be controlled either by the surfaceintegration step or by the bulk-diffusion step, and the crystal faces may be integrated by the spiral mechanism or two-dimensional nucleation mechanism. All of these experimental results are similar to what have been observed in conventional processes of solution crystallization. However, inorganic crystals of millimeter-size cannot re-facet themselves in the GAS process if the seeds are not bounded by well-defined faces.

\section{Notation}

$C_{\mathrm{e}} \quad$ solubility of solute in solutions expanded with carbon dioxide (on a carbon dioxide free basis), $g$ solute $/ 100 \mathrm{~g}$ solvent

$C_{\mathrm{e} 0} \quad$ solubility of solute at an elevated pressure in solutions free of carbon dioxide, $\mathrm{g}$ solute $/ 100 \mathrm{~g}$ solvent

$C_{\mathrm{g}}$ concentration of dissolved carbon dioxide (on a solute-free basis), g carbon dioxide/ $100 \mathrm{~g}$ solvent

$C_{0}$ solubility of solute at atmospheric pressure, g solute/100 g solvent

$\Delta C$ supersaturation of solute, $\mathrm{g}$ solute $/ 100 \mathrm{~g}$ solvent

$P \quad$ pressure, bar

$t$ time of expansion, $h$

$T$ temperature, ${ }^{\circ} \mathrm{C}$

$V_{0} \quad$ volume of original solution, $\mathrm{m}^{3}$

$\alpha \quad$ a factor defined in Eq. (3)

$\varphi$ relative mean square error, $\%$

$\Delta V$ volume increment, $\mathrm{m}^{3}$

\section{Acknowledgements}

The authors gratefully acknowledge the financial support of the National Science Council of the Republic of China.

\section{References}

[1] P.M. Gallagher, M.P. Coffey, V.J. Krukonis, N. Klasutis, ACS Symp. Ser. 406 (1989) 334.

[2] E.M. Berends, O.S.L. Bruinsma, J. de Graauw, G.M. van Rosmalen, in: Proc. 3rd Int. Symp. on Supercritical Fluids (Tome 3), 1994, p. 223.

[3] D.J. Dixon, K.P. Johnston, AIChE J. 1441 (1991) 37.

[4] C.J. Chang, A.D. Randolph, AIChE J. 36 (1990) 939.

[5] A. Kordikowski, C.J. Peters, J. de Swaan Arons, in: Proc. 3rd Int. Symp. on Supercritical Fluids (Tome 3), 1994, p. 217.

[6] C.J. Chang, A.D. Randolph, N.E. Craft, Biotechnol. Progr. 7 (1991) 275 .

[7] P.M. Gallagher, V.J. Krukonis, G.D. Botsaris, AIChE Symp. Ser. 87 (1991) 96.

[8] A. Shishikura, H. Takahashi, S. Hirohama, K. Arai, in: Proc. 2nd Int. Symp. on Supercritical Fluids, 1991, p. 299.

[9] Y. Liou, C.J. Chang, Sep. Sci. Technol. 27 (1992) 1277.

[10] P.M. Gallagher, V.J. Krukonis, L.J. Vandekieft, in: Proc. 2nd Int. Symp. on Supercritical Fluids, 1991, p. 45.

[11] C.Y. Tai, Chuen-Song Cheng, AIChE J., submitted.

[12] A.W. Francis, J. Phys. Chem. 58 (1954) 1099.

[13] J. Nyvlt, O. Sohnel, M. Matuchova, M. Broul, The Kinetics of Industrial Crystallization, Elsevier, New York, 1985, p. 206.

[14] J. Garside, Chem. Eng. Sci. 1425 (1971) 26.

[15] C.Y. Tai, C. Cheng, Y. Huang, J. Crystal Growth 123 (1992) 236.

[16] C.Y. Tai, C. Lin, J. Crystal Growth 82 (1987) 377.

[17] R.D. Doherty, in: B.R. Pamplin (Ed.), Crystal Growth, 2nd ed., Pergamon Press, New York, 1980, p. 485.

[18] R.F. Strickland-Constable, Kinetics and Mechanism of Crystallization, Academic Press, New York, 1968, p. 278.

[19] B. Lewis, in: B.R. Pamplin (Ed.), Crystal Growth, 2nd ed., Pergamon Press, New York, 1980, p. 23.

[20] A.A. Chernov, Modern Crystallography III, Springer, Berlin, 1984, p. 234.

[21] W.J.P.V. Enckevort, Progr. Crystal Growth Characterization 9 (1984) 1. 